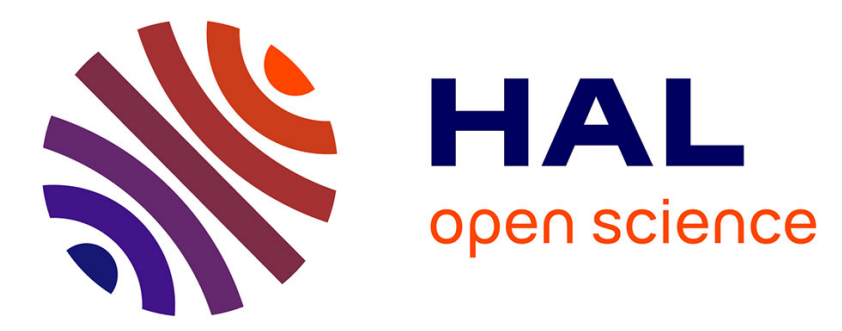

\title{
CYP3A activity influences imatinib response in patients with chronic myeloid leukemia: a pilot study on in vivo CYP3A activity
}

Henrik Gréen, Karin Skoglund, Franz Rommel, Rajaa A. Mirghani, Kourosh Lotfi

\section{To cite this version:}

Henrik Gréen, Karin Skoglund, Franz Rommel, Rajaa A. Mirghani, Kourosh Lotfi. CYP3A activity influences imatinib response in patients with chronic myeloid leukemia: a pilot study on in vivo CYP3A activity. European Journal of Clinical Pharmacology, 2010, 66 (4), pp.383-386. 10.1007/s00228-0090772-y . hal-00553270

\author{
HAL Id: hal-00553270 \\ https://hal.science/hal-00553270
}

Submitted on 7 Jan 2011

HAL is a multi-disciplinary open access archive for the deposit and dissemination of scientific research documents, whether they are published or not. The documents may come from teaching and research institutions in France or abroad, or from public or private research centers.
L'archive ouverte pluridisciplinaire $\mathbf{H A L}$, est destinée au dépôt et à la diffusion de documents scientifiques de niveau recherche, publiés ou non, émanant des établissements d'enseignement et de recherche français ou étrangers, des laboratoires publics ou privés. 


\title{
CYP3A activity influences imatinib response in patients with chronic myeloid leukemia: a pilot study on in vivo CYP3A activity
}

\author{
Henrik Gréen • Karin Skoglund • Franz Rommel • \\ Rajaa A. Mirghani • Kourosh Lotfi
}

Received: 7 September 2009 / Accepted: 26 November 2009 / Published online: 7 January 2010

(C) Springer-Verlag 2009

\begin{abstract}
Purpose Imatinib is currently used for the treatment of chronic myeloid leukemia (CML). The main metabolite CGP74588 has similar potency to that of imatinib and is a product of CYP3A4 and CYP3A5 metabolism. However, the clinical significance of the metabolism on therapeutic response and pharmacokinetics is still unclear. We designed this study to investigate the role of the CYP3A activity in the response to imatinib therapy.

Methods Fourteen CML patients were phenotyped for in vivo CYP3A activity using quinine as a probe drug. The plasma concentration ratio of quinine and its CYP3A metabolite was used for assessing CYP3A activity. The patients were divided into complete molecular responders with undetectable levels of $B C R-A B L$ transcripts after
\end{abstract}

Henrik Gréen and Karin Skoglund contributed equally to this work.

H. Gréen $\cdot$ K. Skoglund $(\bowtie) \cdot K$. Lotfi

Division of Drug Research/Clinical Pharmacology,

Department of Medical and Health Sciences,

Faculty of Health Sciences, Linköping University,

58185 Linköping, Sweden

e-mail: karin.skoglund@liu.se

F. Rommel $\cdot$ K. Lotfi

Department of Hematology, Linköping University Hospital,

Linköping, Sweden

R. A. Mirghani

Division of Clinical Pharmacology,

Department of Laboratory Medicine,

Karolinska University Hospital-Huddinge, Karolinska Institute,

Stockholm, Sweden

Present Address:

R. A. Mirghani

Division of Pharmacology, Faculty of Medicine,

King Fahad Medical City,

Riyadh, Kingdom of Saudi Arabia
12 months of therapy and into partial molecular responders who had failed to achieve a complete molecular response. Results Patients that achieved complete molecular response showed significantly (Mann-Whitney U-test, $p=0.013$ ) higher in vivo CYP3A activity (median quinine metabolic ratio $=10.1)$ than patients achieving partial molecular response (median $=15.9)$.

Conclusions These results indicate a clinical significance of the CYP3A activity and its metabolic products in CML patients treated with imatinib.

Keywords Chronic myeloid leukemia - Imatinib ·

CYP3A4 - CYP3A5 - CGP74588 .

Complete molecular response

\section{Introduction}

Imatinib is a potent BCR-ABL tyrosine kinase inhibitor currently used for the treatment of chronic myeloid leukemia (CML). Pharmacokinetic studies of imatinib in healthy volunteers and patients with CML show that orally administered imatinib is well absorbed and has an absolute bioavailability of $98 \%$ irrespective of the oral dosage form (solution, capsule, tablet) or dosage strength (100 mg, $400 \mathrm{mg}$ ) [1, 2]. The terminal elimination half-life of imatinib is approximately $18 \mathrm{~h} \mathrm{[2],} \mathrm{and} \mathrm{the} \mathrm{main} \mathrm{elimination} \mathrm{pathway} \mathrm{is} \mathrm{via} \mathrm{the}$ biliary-fecal route [3]. In the liver, imatinib is metabolized mainly by cytochrome P450 (CYP) 3A4 and 3A5, although CYP1A1, CYP1A2, CYP1B1, CYP2D6, CYP2C9, and CYP2C19 might also contribute to a minor extent $[4,5]$. The main metabolite in plasma, CGP74588, is mainly formed by CYP3A4 and is present in concentrations of around $20 \%$ of that of the parent drug but with a large interindividual variation. CGP74588 has a longer terminal 
half-life (85-95 h) than imatinib as measured after discontinuation of therapy $[6,7]$. The activity of CYP isoenzymes is known to play an important role in the fate of anticancer agents in patients, but so far there have been relatively few clinical studies that evaluate the influence of drug metabolism on therapeutic outcome. Furthermore, data from various non-oncologic studies have demonstrated a contribution of CYP enzymes to the interindividual variability of drug pharmacokinetics, which is in turn reflected in the variability in therapeutic efficacy and toxicity [8].

Imatinib induces complete molecular responses in the majority of patients with CML in the chronic phase. However, a subgroup of patients is refractory. Unfortunately, in many patients, resistance to imatinib develops a few months or years after starting the treatment [9]. Several mechanisms of resistance have been revealed: mutations or amplification of the target protein, modification of drug disposition, and chromosomal aberrations [8, 9]. Some of the resistance might be overcome by administering a higher dose of imatinib, and Larson et al. showed that a higher plasma level of imatinib correlates with better cytogenetic and molecular responses [6]. In addition, the main CYP3A4 metabolite, CGP74588, is pharmacologically active and has a potency and selectivity similar to those of imatinib [2]. However, the influence of the metabolic pathway and imatinib metabolites on the therapeutic effect of imatinib therapy remains unclear. In this pilot study we investigate the role of the drug metabolizing enzyme activity, i.e., the CYP3A activity in vivo, in the response to imatinib treatment of CML patients.

\section{Materials and methods}

\section{Patient samples}

Fourteen Caucasian CML patients treated with $300-600 \mathrm{mg}$ of imatinib (Glivec) daily were included in the study. Ten patients started on $400 \mathrm{mg} /$ day and two started on 300 and $600 \mathrm{mg}$, respectively. Twelve were in chronic phase and two had reached blast crisis. To assess the in vivo CYP3A activity at inclusion, the patients were given a $250 \mathrm{mg}$ quinine tablet (Kinin NM Pharma); $16 \mathrm{~h}$ later a blood sample was collected and the plasma concentration of quinine and 3-hydroxyquinine was measured using HPLC as previously described $[10,11]$. Thereafter, the quinine/3hydroxyquinine ratio was determined to estimate the activity of CYP3A. Five patients were phenotyped before the start of imatinib therapy and after 2 months (range 1-6 months) following the same procedure. Four patients were phenotyped during imatinib therapy and five patients were phenotyped after imatinib therapy was stopped. None of the patients were co-treated with known CYP3A4 inhibitors or inducers at the time for CYP3A phenotyping. Patient blood status was followed weekly during the first month of imatinib therapy and then every third month of the first year. Leukopenia was evaluated according to the Common terminology criteria for adverse events (CTCAE) version 3.0 and correlated to CYP3A activity. For evaluation of thrombocytopenia, CTCAE grading and the percentage of maximum decrease in platelets compared to baseline were used for correlation to the CYP3A activity. The molecular response to imatinib therapy was evaluated using $B C R-A B L /$ glucuronidase-beta (GUS) ratios and the patients were divided into complete molecular responders (CMRs) within 12 months and partial molecular responders (PMRs) who had failed to achieve a CMR status within 12 months. CMR status was defined as undetectable levels of $B C R-A B L$ transcripts. Written informed consent was obtained from all patients before inclusion, and the study was approved by the Regional Human Ethics Committee in Linköping, Sweden.

\section{Chemosensitivity assay}

The chemosensitivities to imatinib and its main metabolite CGP74588 (kindly supplied by Novartis, Basel, Switzerland) were assessed using the MTT assay as previously described [12]. In brief, the chronic myeloid leukemia cell line K562 [American Type Culture Collection (ATCC), Manassas, VA, USA] were seeded in 96-well plates $(100 \mu \mathrm{L} /$ well, $2 \times 10^{5}$ cells $/ \mathrm{mL}$ ) and incubated for $72 \mathrm{~h}$ in the absence or presence of nine different drug concentrations in triplicate. Post drug exposure, $10 \mu \mathrm{L} /$ well of MTT solution $[5 \mathrm{mg} / \mathrm{mL}$ 3-(4,5-dimethylthiazol-2-yl)-2,5-diphenyltetrazolium bromide] was added, and the cells were re-incubated for $4 \mathrm{~h}$ at $37^{\circ} \mathrm{C}$. To dissolve the formed formazan salt crystals, $100 \mu \mathrm{L}$ of $10 \%$ SDS in a $10 \mathrm{mM} \mathrm{HCl}$ solution were added followed by incubation overnight at $37^{\circ} \mathrm{C}$. The absorbance was measured at $580 \mathrm{~nm}$ in a plate reader (Synergy HT, Bio-Tek, USA) and the chemosensitivity was expressed as $\mathrm{IC}_{50}$.

\section{Statistical analysis}

The nonparametric Mann-Whitney U test and GraphPad Prism 4.0 software (GraphPad Software, San Diego, CA, USA) were used to analyze the difference in CYP3A activity between CMRs and PMRs. A statistical difference with a $p$-value less than 0.05 was considered significant.

\section{Results}

After 12 months of imatinib therapy, six patients (five males and one female, median age 41 years, range 20-53) had achieved CMR and eight patients (three males and five females, median age 65 years, range 39-79) had only achieved PMR status. Median values of the prognostic 
Hasford score [13] were 1059 and 791 in the CMR and PMR groups, respectively. CMRs showed significantly $(p=0.013)$ higher CYP3A activity (low quinine ratio, median $=10.1$ ) than PMRs (high quinine ratio, median = 15.9) as presented in Fig. 1. The correlation between CYP3A activity and molecular response is still statistically significant after excluding the two patients in blast crisis. Five patients were assessed again for CYP3A activity after 2 months of imatinib therapy. Three of these patients showed no change in CYP3A activity, one CMR patient had an increased activity and one PMR patient had decreased activity (Fig. 2), indicating that imatinib does not greatly alter CYP3A activity. However, it is not known whether intraindividual CYP3A4 activity is changed upon long-term imatinib therapy, although it has been shown that CYP3A4 inducers and inhibitors may alter the pharmacokinetics of imatinib [14-16].

The MTT data demonstrate potencies in the same range for imatinib $\left(\mathrm{IC}_{50}=0.32 \mu \mathrm{M}, \mathrm{n}=3, \mathrm{CV}=12 \%\right)$ and its main metabolite $\left(\mathrm{IC}_{50}=0.91 \mu \mathrm{M}, \mathrm{n}=3, \mathrm{CV}=15 \%\right)$, as demonstrated by others [2]. Four patients experienced grade 1-2 leukopenia and two patients suffered grade 3-4. The decrease in platelets in the patients ranged from 5 to $94 \%$, and two patients suffered grade 4 thrombocytopenia during the first year of therapy. Neither leukopenia nor thrombocytopenia could be significantly correlated to CYP3A activity in this material. For three patients imatinib therapy was omitted before the end of the first year. One patient had a blast crisis after 8 months of imatinib and received stem cell transplantation. Two patients had to stop imatinib therapy due to adverse events after 1 and 2 months of therapy, respectively. The adverse events were

$$
p=0.013
$$

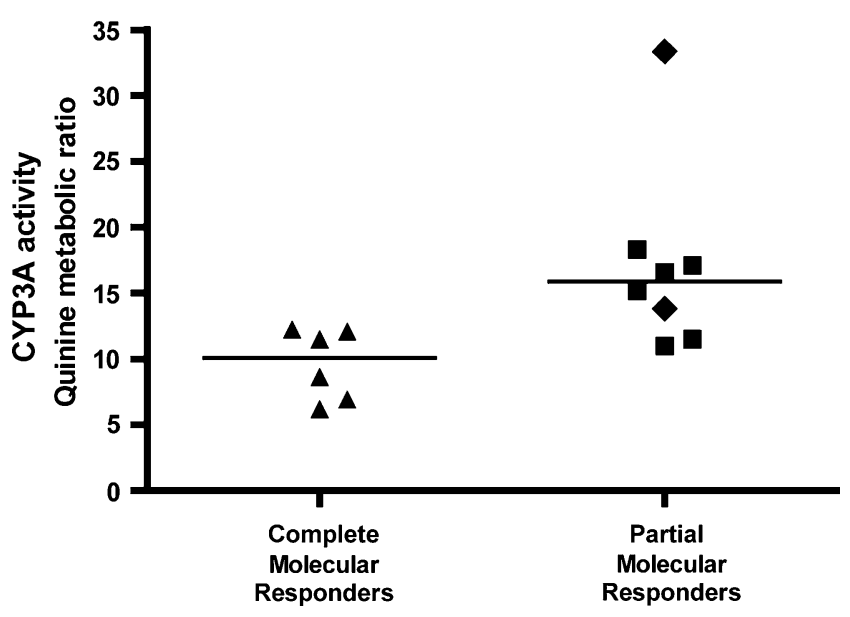

Fig. 1 The effect of in vivo CYP3A activity on the response to imatinib treatment. The CYP3A activity was determined by measuring the metabolic ratio of quinine/3-OHquinine (low ratio $=$ high activity). The patients' molecular response was evaluated after 12 months and the patients were grouped into complete molecular responders and partial molecular responders. The line represents the median activity in each group. Two patients had reached blast crisis when starting imatinib therapy; they are shown as diamonds in the figure

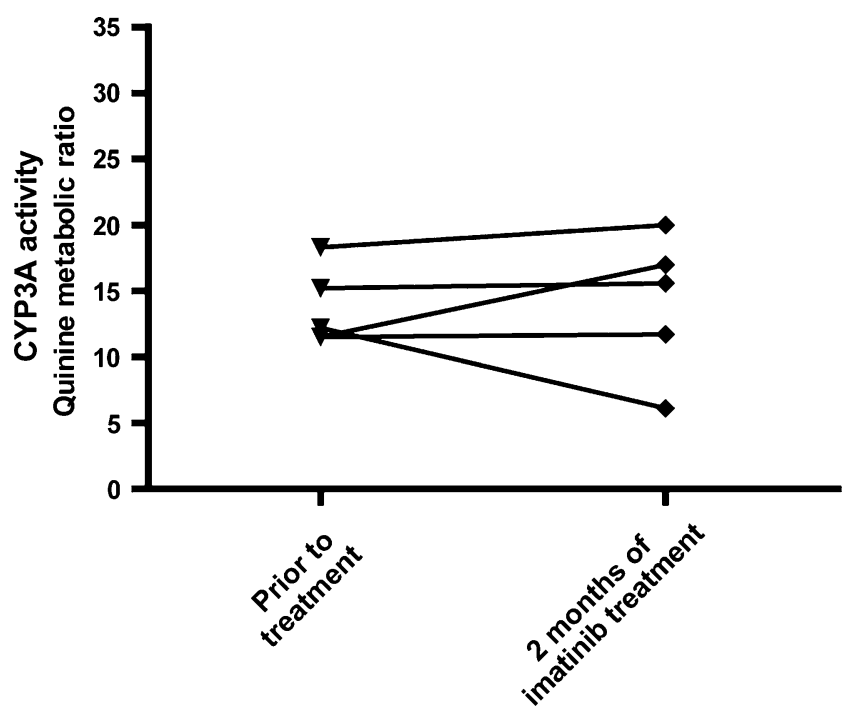

Fig. 2 The induction/decrease in in vivo CYP3A activity after 2 months of imatinib therapy

skin rash and hepatotoxicity, and these patients had low CYP3A activity with a quinine metabolic ratio of 33.3 and 16.6 , respectively. Some patients also had shorter discontinuations of therapy or changed dosing due to adverse events.

\section{Discussion}

These results indicate that the metabolic CYP3A activity is important for the response of imatinib in CML patients. It is noteworthy that CML patients with high CYP3A activity (probably leading to a higher amount of metabolite) respond better to imatinib therapy than patients with low activity. Recently, the plasma concentration of imatinib was shown to be important for the response [6], and our results indicate that the effect and potency of the metabolites might also be of clinical importance. One explanation for the importance of the CYP3A activity might be that CGP74588 or other CYP3A products of imatinib are active against sequence variations of the $B C R-A B L$ that are not affected by imatinib. We know that the main metabolite is present in concentrations of around $20 \%$ of that of the parent drug after 1 month of therapy, but with a large interindividual variation that might be explained by differences in CYP3A activity $[6$, 7]. The metabolite also has a long terminal half-life (85-95 h) $[6,7]$, indicating that it might reach steady state and contribute to the clinical effects at a later time point during therapy. The plasma concentration of the metabolite is also above the effective concentration of the substances in an in vitro setting [7], indicating that the metabolite might have clinical significance. Rochat et al. have shown that several other metabolites than CGP74588 are present in plasma at concentrations equal to or in the same range as the main 
metabolite. Some of these metabolites have also been shown to be CYP3A4 products in vitro [5]. If any of these are pharmacologically active they might contribute to the effect of CYP3A activity on the therapeutic response reported here. It should be noted that the number of patients in this study is small and that the study should be regarded as a pilot study. There might be a number of confounding factors such as the fact that patients were in different disease phases or that some patients were phenotyped during imatinib therapy; other factors might also influence imatinib pharmacokinetics such as body weight and $\alpha$-acid glycoprotein plasma levels $[4,6$, $17,18]$.

\section{Conclusions}

This study indicates that the CYP3A activity correlates with the tumor response after imatinib therapy and patients with higher CYP3A activity seem to respond better to imatinib. Although the study is small in scale, the results indicate that CYP3A activity is important for the effect of imatinib. It is known from the IRIS and CML91 trials that patients who achieve complete cytogenetic response by 12 months have a higher survival rate compared to patients who do not achieve complete cytogenetic response [19]. It has also been shown that patients not achieving an early response to therapy are at higher risk for treatment failure and have lower chances of eventually achieving a complete cytogenetic response or major molecular response [20]. Clinically, it would therefore be advantageous to identify patients who will not respond as quickly a priori since they may benefit from more aggressive or alternative therapy. Determining the CYP3A activity in CML patients prior to treatment might be a way to individualize imatinib dosing in the future. However, results from larger studies are needed before the influence of the CYP3A activity can be definitively determined.

Acknowledgments This work was supported by grants from the Swedish Cancer Society, the Swedish Research Council - Medicine (3902), the Signe and Olof Wallentin Foundation, the County Council of Östergötland, Medical Research Council of Southeast Sweden, Capio's Research Foundation, and the Swedish Society of Medicine.

\section{References}

1. Peng B, Dutreix C, Mehring G, Hayes MJ, Ben-Am M, Seiberling $\mathrm{M}$ et al (2004) Absolute bioavailability of imatinib (Glivec) orally versus intravenous infusion. J Clin Pharmacol 44:158-162

2. Cohen MH, Williams G, Johnson JR, Duan J, Gobburu J, Rahman A et al (2002) Approval summary for imatinib mesylate capsules in the treatment of chronic myelogenous leukemia. Clin Cancer Res 8:935-942

3. Gschwind HP, Pfaar U, Waldmeier F, Zollinger M, Sayer C, Zbinden $\mathrm{P}$ et al (2005) Metabolism and disposition of imatinib mesylate in healthy volunteers. Drug Metab Dispos 33:15031512

4. Peng B, Lloyd P, Schran H (2005) Clinical pharmacokinetics of imatinib. Clin Pharmacokinet 44:879-894

5. Marull M, Rochat B (2006) Fragmentation study of imatinib and characterization of new imatinib metabolites by liquid chromatography-triple-quadrupole and linear ion trap mass spectrometers. J Mass Spectrom 41:390-404

6. Larson RA, Druker BJ, Guilhot F, O'Brien SG, Riviere GJ, Krahnke T et al (2008) Imatinib pharmacokinetics and its correlation with response and safety in chronic-phase chronic myeloid leukemia: a subanalysis of the IRIS study. Blood 111:4022-4028

7. le Coutre P, Kreuzer KA, Pursche S, Bonin M, Leopold T, Baskaynak G et al (2004) Pharmacokinetics and cellular uptake of imatinib and its main metabolite CGP74588. Cancer Chemother Pharmacol 53:313-323

8. Rochat B (2005) Role of cytochrome P450 activity in the fate of anticancer agents and in drug resistance: focus on tamoxifen, paclitaxel and imatinib metabolism. Clin Pharmacokinet 44:349-366

9. Engelman JA, Settleman J (2008) Acquired resistance to tyrosine kinase inhibitors during cancer therapy. Curr Opin Genet Dev 18:73-79

10. Mirghani RA, Hellgren U, Westerberg PA, Ericsson O, Bertilsson L, Gustafsson LL (1999) The roles of cytochrome P450 3A4 and $1 \mathrm{~A} 2$ in the 3-hydroxylation of quinine in vivo. Clin Pharmacol Ther 66:454-460

11. Mirghani RA, Ericsson O, Cook J, Yu P, Gustafsson LL (2001) Simultaneous determination of quinine and four metabolites in plasma and urine by high-performance liquid chromatography. J Chromatogr B Biomed Sci Appl 754:57-64

12. Green H, Lotfi K, Zackrisson AL, Peterson C (2003) Spontaneous reversal of p-glycoprotein expression in multidrug resistant cell lines. Pharmacol Toxicol 93:297-304

13. Hasford J, Pfirrmann M, Hehlmann R, Allan NC, Baccarani M, Kluin-Nelemans JC et al (1998) A new prognostic score for survival of patients with chronic myeloid leukemia treated with interferon alfa. Writing Committee for the Collaborative CML Prognostic Factors Project Group. J Natl Cancer Inst 90:850-858

14. Frye RF, Fitzgerald SM, Lagattuta TF, Hruska MW, Egorin MJ (2004) Effect of St John's wort on imatinib mesylate pharmacokinetics. Clin Pharmacol Ther 76:323-329

15. Bolton AE, Peng B, Hubert M, Krebs-Brown A, Capdeville R, Keller U et al (2004) Effect of rifampicin on the pharmacokinetics of imatinib mesylate (Gleevec, STI571) in healthy subjects. Cancer Chemother Pharmacol 53:102-106

16. Dutreix C, Peng B, Mehring G, Hayes M, Capdeville R, Pokorny $\mathrm{R}$ et al (2004) Pharmacokinetic interaction between ketoconazole and imatinib mesylate (Glivec) in healthy subjects. Cancer Chemother Pharmacol 54:290-294

17. Petain A, Kattygnarath D, Azard J, Chatelut E, Delbaldo C, Geoerger B et al (2008) Population pharmacokinetics and pharmacogenetics of imatinib in children and adults. Clin Cancer Res 14:7102-7109

18. Gambacorti-Passerini C, Zucchetti M, Russo D, Frapolli R, Verga M, Bungaro $S$ et al (2003) Alpha1 acid glycoprotein binds to imatinib (STI571) and substantially alters its pharmacokinetics in chronic myeloid leukemia patients. Clin Cancer Res 9:625-632

19. Roy L, Guilhot J, Krahnke T, Guerci-Bresler A, Druker BJ, Larson RA et al (2006) Survival advantage from imatinib compared with the combination interferon-alpha plus cytarabine in chronic-phase chronic myelogenous leukemia: historical comparison between two phase 3 trials. Blood 108:1478-1484

20. Quintas-Cardama A, Kantarjian H, Jones D, Shan J, Borthakur G, Thomas D et al (2009) Delayed achievement of cytogenetic and molecular response is associated with increased risk of progression among patients with chronic myeloid leukemia in early chronic phase receiving high-dose or standard-dose imatinib therapy. Blood 113:6315-6321 